\title{
Diagnostic evaluations of ultrasound and magnetic resonance imaging in mammary duct ectasia and breast cancer
}

\author{
LEI SONG ${ }^{1 *}$, LIANG LI $^{2 *}$, BIN LIU $^{3}$, DEXIN YU ${ }^{4}$, FENGGUO SUN $^{5}$, MINGMING GUO $^{2}$, \\ ZHENGMIN RUAN $^{6}$ and FEIXUE ZHANG $^{6}$
}

\author{
${ }^{1}$ Equipment Division; ${ }^{2}$ Department of Breast Surgery, The Second Hospital of Shandong University; ${ }^{3}$ Department of \\ Radiology, The First Clinical College, Shandong University of Traditional Chinese Medicine; ${ }^{4}$ Department of Radiology, \\ Qilu Hospital, Shandong University; ${ }^{5}$ Department of Radiology, Division of MRI; ${ }^{6}$ Department of Radiology, \\ Division of Ultrasound, The Second Hospital of Shandong University, Jinan, Shandong 250033, P.R. China
}

Received January 12, 2017; Accepted October 24, 2017

DOI: 10.3892/ol.2017.7514

\begin{abstract}
The objective of the present study was to evaluate the diagnostic efficiency of ultrasound (US) and magnetic resonance imaging (MRI) in the diagnosis and differential diagnosis of mammary duct ectasia (MDE) and breast cancer. This retrospective study was performed on 35 patients with MDE and 105 patients with breast cancer using US and MRI. Imaging features, semi-quantitative and quantitative parameters were analyzed to determine their diagnostic value for MDE and breast cancer. The average age of patients with breast cancer was increased compared with that of patients with MDE. There were no significant differences in local packages with or without tenderness ratio $(\mathrm{P}=0.259)$ and grade of color Doppler flow imaging $(\mathrm{P}=0.273)$ between the two groups. However, the morphological changes were significantly increased in breast cancer compared with MDE. In addition, there were significant diagnostic differences in US and MRI between breast cancer and MDE, including resistance index, US elastography, time-signal intensity curve, apparent diffusion coefficient, early-stage enhancement ratio, peak-of-enhancement ratio and $\mathrm{T}_{\text {peak }}(\mathrm{P}<0.05)$. However, there were no observable significant diagnostic differences between US, MRI and US with MRI for MDE and breast cancer $(\mathrm{P}=0.103, \mathrm{P}=0.263$ and $\mathrm{P}=0.403$ respectively). Diagnosis of MDE and breast cancer requires full evaluation of multiple parameters and morphological changes of US and MRI to increase the diagnostic efficiency. US, MRI and US with MRI were all of diagnostic value for
\end{abstract}

Correspondence to: Professor Feixue Zhang, Department of Radiology, Division of Ultrasound, The Second Hospital of Shandong University, 247 Beiyuan Street, Jinan, Shandong 250033, P.R. China

E-mail: snow-1860@163.com

${ }^{*}$ Contributed equally

Key words: mammary duct ectasia, breast cancer, ultrasound, magnetic resonance imaging, case-control study
MDE and breast cancer, while US with MRI had the highest efficacy.

\section{Introduction}

Mammary duct ectasia (MDE) is a type of non-puerperal and non-specific inflammation (1). Occasionally, MDE presents with similar clinical features to those of breast cancer (2). For example, MDE often presents with an atypical and insidious mass without inflammation (3), rendering it difficult to diagnose and differentiate from breast cancer (4). Furthermore, breast cancer may be confused with benign lesion in imaging, particularly inflammatory cancer, which had an incidence rate of between 1 and 6\% in the United States between 1988 and 2000 (5) and resembles MDE and breast abscess (5). Therefore, it is important to differentiate between MDE and breast cancer for timely diagnosis and subsequent treatment.

Breast cancer may be diagnosed using ultrasound (US) and magnetic resonance imaging (MRI) technology (6-8). However, to the best of our knowledge, there is no report on the detailed parameter comparisons between US and MRI in the differential diagnosis of MDE and breast cancer. For example, the diagnostic value of dynamic contrast-enhanced MRI (DCE-MRI) for MDE has been seldom discussed (9). Therefore, in the present study, patients with suspected MDE and breast cancer were recruited. The diagnostic values of US and MRI were evaluated.

\section{Materials and methods}

Patients. Patients with suspected MDE and breast cancer were recruited between May 2012 and December 2015 in The Second Hospital of Shandong University (Jinan, China). All patients were examined using US and MRI. Mammotome biopsy or resection was performed a week after the imaging examinations. The final diagnosis was made by pathological analysis (H\&E staining).

Patients were included if they were: i) female; ii) clinically suspected of presenting with MDE or breast cancer by physical examination; and iii) consented to participate in the present study. Patients were excluded if they: i) declined to join in the 
research; ii) declined to be examined by US and MRI; iii) were unable to be examined using MRI due to implanted metal; iv) had been treated with neoadjuvant chemotherapy and/or needle biopsy; v) refused mammotome biopsy or resection; or vi) presented with other types of malignancy. The Medical Ethics Board of The Second Hospital of Shandong University approved the present study. Written informed consent was obtained from all patients or their families.

$H \& E$ staining. H\&E staining was performed according to routine procedure. Briefly, tissues were fixed with $10 \%$ neutral formalin at room temperature for $24 \mathrm{~h}$, embedded in paraffin and cut into $4 \mu \mathrm{m}$ sections. Then tissue sections were dewaxed in xylene and rehydrated in graded alcohols at room temperature. Following washing with running water and distilled water, sections were stained with hematoxylin for 3-5 min at room temperature. Following washing again with running water, sections were differentiated with $1 \% \mathrm{HCl}$ in $70 \%$ alcohol for 5-10 sec at room temperature. Then sections were stained with eosin for 1-4 min at room temperature following washing with running water. Subsequent to dehydration and differentiation in alcohol at room temperature, sections were mounted and observed under light microscopy (OLYMPUS BX45; Olympus Corporation, Tokyo, Japan). The magnification was 400x.

US. US examination was performed using LOGIQ E9 ultrasound instrument (GE Healthcare Life Sciences, Little Chalfont, UK). Patients were fully exposed in a supine position with the arms raised above the head. A 9-14 MHz high-frequency probe was used to bilaterally scan the breasts and axillaries of the patients. US elastography (UE) was performed following routine US.

Routine US features (10) were classified into four types: Mammary-duct dilation (type I), cyst-and-solid mass (type II), solid mass (type III) and abscess (type IV). A five-point scale was determined based on the color of nodules, which was assessed by eye, in UE $(11,12)$ : Primarily green scored 1 point; blue center and predominantly green surrounding scored 2 points; equal amount of green and blue scored 3 points; predominately blue or mixed with small proportion of green scored 4 points; surrounding tissues of mostly blue scored 5 points. A lesion with scores of 1 to 3 points was considered benign, otherwise was malignant.

Two-dimensional images, color Doppler flow imaging (CDFI) and elastography were also recorded and analyzed according to Breast Imaging-Reporting and Data System (BI-RADS) lexicon of the American College of Radiology (ACR) (13). All data were collected and analyzed by two US specialists (Department of Radiology, Division of Ultrasound, the Second Hospital of Shandong University) who were blinded to the final pathological results. Disagreement was settled either by consensus or a third reviewer (Department of Radiology, Division of Ultrasound, the Second Hospital of Shandong University) blinded to the final pathological results.

MRI. MRI examination was performed using a Signa HD x3.0T TWINSP MR system (GE Healthcare Life Sciences). Patients were in a prone position. MRI plain scanning was performed from the direction of the feet using the parameters of Ax short tau inversion recovery (STIR; TR 8200/TE 36,
Matrix 512x512, NEX 2.00), Ax T1 fast spin echo (FSE; TR 40/TE 7.9, NEX 2.00, Matrix 512x512), bilateral sag (TR3600/TE110, Matrix 512x512, NEX 2.00) and diffusion-weighted imaging ( $b=0,1,000$; TR6000/TE70, Matrix $512 \times 512$, NEX 4.00). DCE-MRI scanning was performed by injecting the contrast medium Gadolinium (Gd)-diethylene triamine pentacetate acid (Beilu Pharmaceutical Co., Ltd., Beijing, China) at a dosage of $0.2 \mathrm{mmol} / \mathrm{kg}$ in $20 \mathrm{ml}$ saline and a speed of $1.5 \mathrm{ml} / \mathrm{sec}$ into the dorsum manus vein, followed by another $20 \mathrm{ml}$ saline. A scanning mask was obtained prior to contrast medium injection. The 3-dimensional vibrant-axial scanning was performed simultaneously with the injection of the contrast medium (parameters: TR 4.3/TE 2.1, Matrix $512 \times 512$, NEX 0.7) for eight phases. Subsequently, 3-dimensional vibrant-sagittal scanning was performed (parameters: TR 4.3/TE 1.8, Matrix 512x512, NEX 0.7). Liquefied and necrotic areas were avoided and, in the region of interest (ROI) of apparent diffusion coefficient (ADC), the solid part was identified and measured 3 times. The area of the ROI was $\geq 2 \mathrm{~mm}^{2}$.

All data were processed offline on an AW Volume shared by 2 workstations (GE Healthcare Life Sciences) and independently interpreted by two senior MRI experts (Department of Radiology, Division of MRI, the Second Hospital of Shandong University) blinded to the final pathological results. The morphological features of the lesions were analyzed according to BI-RADS lexicon of the ACR (13).

The measurement parameters included early-stage enhancement ratio (EER), peak-of-enhancement ratio (PER), time peak (T peak) and time-signal intensity curve (TIC). The formula for EER calculation was: EER $=S_{1}-S_{0} / S_{0} \times 100 \% ; S_{1}$ is the signal intensity obtained $1 \mathrm{~min}$ after contrast medium injection and $S_{0}$ is the signal intensity obtained prior to injection. The formula for PER calculation was: $P E R=S_{\text {peak }}-S_{0} / S_{0} \times 100 \%$; $S_{\text {peak }}$ represented the peak signal intensity obtained prior to or following contrast medium injection; $S_{0}$ represented the signal intensity obtained prior to injection. $\mathrm{T}$ peak represented the time between contrast medium injection and the highest signal intensity. TIC was classified into 3 types (14): Persistently enhancing (type I), plateau (type II) and washout (type III).

Statistical analysis. All data were analyzed using SPSS software (version 17.0; SPSS, Inc., Chicago, IL, USA). Measurement data were presented as the mean \pm standard deviation. Quantitative data are presented as percentages. The difference between MDE and breast cancer was compared using Chi-square test and analysis of the sensitivity, specificity, accuracy, positive and negative predictive value. $\mathrm{P}<0.05$ was considered to indicate a statistically significant difference.

\section{Results}

Clinical features. A total of 35 cases of MDE and 105 cases of breast cancer were diagnosed by pathology. All patients were female. Clinical features differed between the MDE and breast cancer groups (Table I). The mean age was $33.58 \pm 8.81$ (range, 23-55) years for patients with MDE and was $44.97 \pm 11.04$ (range, 17-78) years for patients with breast cancer. For the 35 patients with MDE, the duration of symptoms prior to diagnosis ranged from 4 days to 2 years. 
Table I. Clinical features of included patients.

\begin{tabular}{|c|c|c|c|c|c|c|c|c|c|}
\hline Variables & $\begin{array}{l}\text { Age, } \\
\text { years }\end{array}$ & $\begin{array}{c}\text { Local } \\
\text { masses }\end{array}$ & $\begin{array}{l}\text { Number } \\
\text { of masses }\end{array}$ & $\begin{array}{c}\text { Skin } \\
\text { swelling }\end{array}$ & $\begin{array}{c}\text { Skin } \\
\text { ulceration }\end{array}$ & $\begin{array}{c}\text { Nipple } \\
\text { inversion }\end{array}$ & $\begin{array}{c}\text { Nipple } \\
\text { discharge }\end{array}$ & Menstruation & Menopause \\
\hline MDE & $.58 \pm 8.81$ & $31(88.6 \%)$ & $4(11.4 \%)$ & $8(22.9 \%)$ & $7(20 \%)$ & $10(28.6 \%)$ & $12(34.3 \%)$ & 33 & $\%)$ \\
\hline Breast cancer & $44.97 \pm 11.04$ & $99(94.3 \%)$ & $6(5.7 \%)$ & $4(3.8 \%)$ & $10(9.5 \%)$ & $14(13.3 \%)$ & 0 & $75(71.4 \%)$ & $30(28.6 \%)$ \\
\hline
\end{tabular}

MDE, mammary duct ectasia.

Table II. Comparisons of morphological features on US between MDE and breast cancer.

\begin{tabular}{|c|c|c|c|c|c|c|c|c|}
\hline \multirow[b]{2}{*}{ Variables } & \multicolumn{2}{|c|}{ Ratio } & \multicolumn{2}{|c|}{ Border } & \multicolumn{2}{|c|}{ Morphology } & \multicolumn{2}{|c|}{ Microcalcification } \\
\hline & $>1$ & $<1$ & Clear & Unclear & Regular & Irregular & Present & Absent \\
\hline MDE (no. cases) & 0 & 35 & 19 & 16 & 22 & 13 & 0 & 35 \\
\hline Breast cancer (no. cases) & 31 & 74 & 97 & 8 & 5 & 100 & 48 & 57 \\
\hline$\chi^{2}$ value & 13.27 & & 36.72 & & 56.91 & & 24.35 & \\
\hline P-value & $<0.001$ & & $<0.001$ & & $<0.001$ & & $<0.001$ & \\
\hline
\end{tabular}

US, ultrasound; MDE, mammary duct ectasia.

Local masses with and without tenderness in the breast were the major clinical manifestations and were identified in 31 $(88.6 \%)$ patients. Tenderness without masses was identified in $4(11.4 \%)$ patients. Skin swelling was observed in $8(22.9 \%)$ patients, 7 (20\%) patients exhibited skin ulceration with sinus formation, $10(28.6 \%)$ patients exhibited nipple inversion (bilateral in 1 patient) and $12(34.3 \%)$ patients experienced nipple discharge. A number of patients experienced multiple symptoms. A total of $31(88.6 \%)$ patients were married with a history of childbirth and $4(11.4 \%)$ patients had no history of marriage or childbirth. Normal menstruation was observed in $33(94.3 \%)$ patients and $2(5.7 \%)$ patients were menopausal. No patients had a history of smoking, hepatitis, diabetes, tuberculosis or a family history of breast cancer.

For the 105 patients with breast cancer, the duration of symptoms prior to diagnosis ranged from 2 days to 6 months. Local masses with and without tenderness in the breast were the major clinical manifestations, observed in $99(94.3 \%)$ patients. Tenderness without masses was identified in $6(5.7 \%)$ patients. Skin swelling was observed in 4 (3.8\%) patients, $10(9.5 \%)$ patients exhibited skin ulceration with sinus formation (1 patient exhibited skin ulceration without sinus formation) and $14(13.3 \%)$ patients exhibited nipple inversion. No patients exhibited nipple discharge. A total of 104 patients had married and only 1 patient had no history of marriage. Normal menstruation was observed in 75 (71.4\%) patients and $30(28.6 \%)$ patients were in menopause. No patients had a history of smoking. A total of 4 patients had a history of hepatitis, 4 patients had diabetes and 10 patients had a family history of breast cancer.

The mean age of patients with breast cancer was increased compared with that of patients with MDE. However, there were no observable differences of local mass with or without tenderness between the two groups by chi-square test $(\mathrm{P}=0.259)$.

Analysis of MDE and breast cancer with US. US was performed to compare the difference between MDE and breast cancer. The morphological features of MDE and breast cancer were analyzed and summarized in Table II. Of the 35 cases of MDE, 19 (54.3\%) presented with a clear border (Fig. 1A) and weak blood signals (Fig. 1B) and 16 (45.7\%) with an obscured border. Morphology was regular in 22 cases $(62.9 \%)$ and irregular in $13(37.1 \%)$. There was no aspect ratio $>1$ or calcification. The 105 cases of breast cancer exhibited an aspect ratio $>1$ with or without posterior attenuation in 31 cases $(29.5 \%)$, obscure boundary (including spicule sign, sharp corner sign and crab claws sign) in 97 cases $(92.4 \%)$, clear border in 8 (7.6\%) cases, irregular morphology in 100 cases $(95.2 \%)$, regular morphology in $5(4.8 \%)$ cases, and microcalcification/clustered calcification in $48(45.7 \%)$ cases. The difference in morphological features on US between MDE and breast cancer was significant $(\mathrm{P}<0.05)$.

Among the 35 MDE cases, 28 were classified as CDFI 0-1 grading and 7 as CDFI 2-3 grading (Table III). Among breast cancer cases, 74/105 were classified as CDFI 0-1 grading and 31/105 were classified as CDFI 2-3 grading. There was no statistical difference observed between the two groups in CDFI grading by chi-square test $\left(\chi^{2}=1.20, \mathrm{P}=0.273\right)$.

In 35 cases of MDE, resistance index (RI) was 0.52-0.86 (mean, 0.593 \pm 0.042 ), including 26 cases of RI $\leq 0.7$ and 9 cases $>0.7$. In 105 cases of breast cancer, RI was 0.48-0.89 (mean, $0.688 \pm 0.086$ ), including 51 cases of RI $\leq 0.7$ and 54 cases $>0.7$. There was a statistically significant difference between the two groups in RI by chi-square test $\left(\chi^{2}=7.013\right.$, $\mathrm{P}=0.008, \mathrm{P}<0.05)$. 
Table III. Comparisons of CDFI grading, RI and UE between MDE and breast cancer.

\begin{tabular}{|c|c|c|c|c|c|c|}
\hline \multirow[b]{2}{*}{ Variables } & \multicolumn{2}{|c|}{ CDFI grading } & \multicolumn{2}{|c|}{ RI } & \multicolumn{2}{|c|}{ UE } \\
\hline & $0-1$ & $2-3$ & $>0.7$ & $\leq 0.7$ & $1-3$ & $4-5$ \\
\hline MDE (no. cases) & 28 & 7 & 9 & 26 & 33 & 2 \\
\hline Breast cancer (no. cases) & 74 & 31 & 54 & 51 & 15 & 90 \\
\hline$\chi^{2}$ & 1.20 & & 7.013 & & 74.56 & \\
\hline P-value & 0.273 & & 0.008 & & $<0.001$ & \\
\hline
\end{tabular}

CDFI, color Doppler flow imaging; RI, resistance index; UE, ultrasound elastography; MDE, mammary duct ectasia.
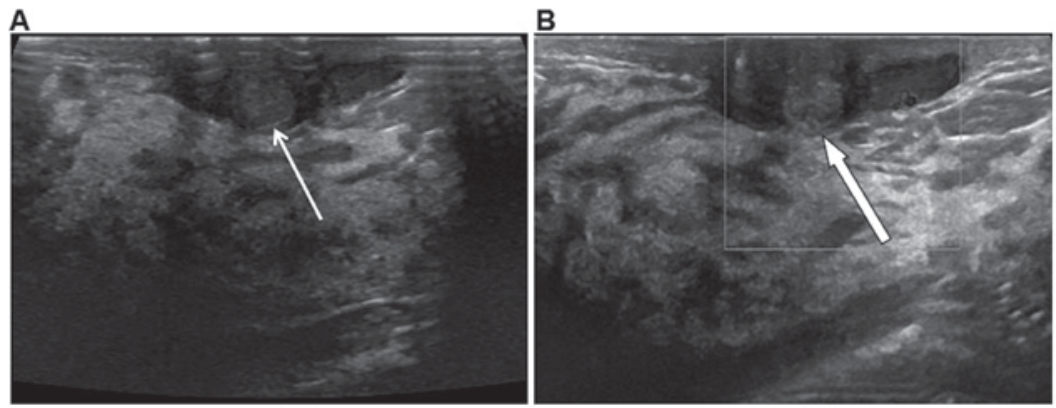

Figure 1. Ultrasound features of mammary duct ectasia. (A) Ultrasound-identified pipe-like hypo echo under the right nipple (arrow) with clear border. (B) Color Doppler flow imaging identified weak blood signals. Arrow indicates the thick dotted echo and cloddy hyper echo.
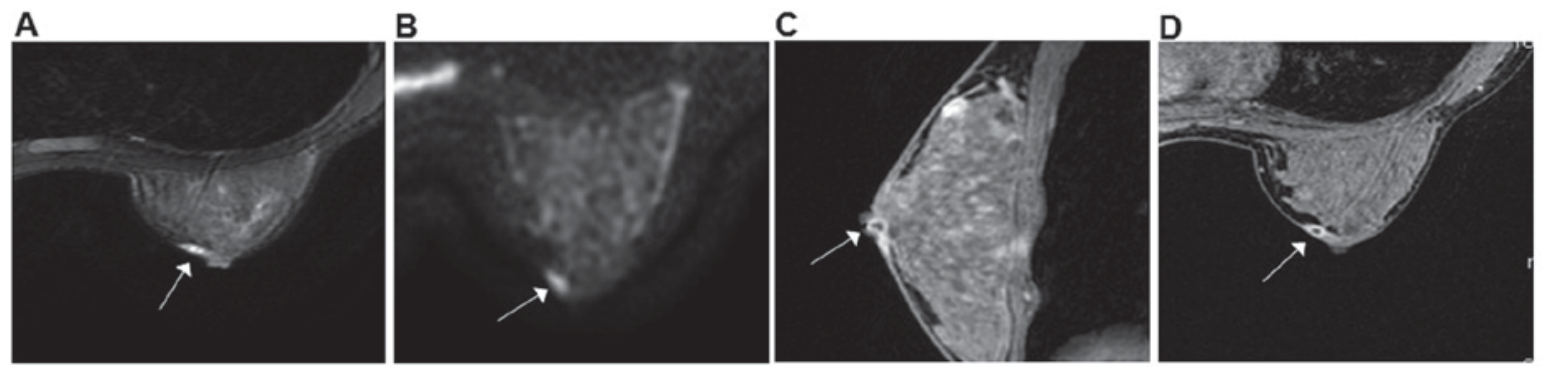

Figure 2. MRI features of MDE. (A) MRI diffusion weighted imaging sequencing identified a strip hyper echo under the right nipple with unclear border (arrow). (B) Short tau inversion recovery sequence identified an uneven strip hyper echo with clear border (arrow). (C) DCE-MRI (sagittal view). The arrow indicates that the lesion was enhanced circularly. (D) DCE-MRI (axial view) revealed that the lesion was enhanced circularly (arrow). There was no enhancement in the delayed phase. MRI and pathology diagnosed MDE. MRI, magnetic resonance imaging; MDE, mammary duct ectasia; DCE, dynamic contrast enhanced.

In 35 MDE cases, 33 had a UE score of 1-3 and 2 had a score of 4 and 5. The mean UE score for a patient with MDE was $2.31 \pm 0.83$. In 105 breast cancer cases, 15 had a UE score of 1-3 and 90 had a score of 4 and 5. The mean UE score for patients with breast cancer was $3.80 \pm 0.69$. There was a statistical difference between the two groups of UE by chi-square test $(\mathrm{P}<0.001)$, which indicated that UE was useful to distinguish between MDE and breast cancer.

Analysis of MDE and breast cancer with MRI. MRI was performed to compare MDE and breast cancer. MDE and breast cancer exhibited heterogeneous high signal in STIR sequence and homogeneous or heterogeneous high, low, mixed signal in T1FSE sequence on plain scan with MRI. Diffusion weighted imaging sequencing identified heterogeneous high signal. The morphology of lesions was irregular with clear or unclear boundary. The features of plain scan of MRI and morphology were unspecific.

Of 35 cases, DCE-MRI of MDE revealed that 26 lesions exhibited thickened wall, resembling an enhanced ring, duct or clumps (Fig. 2); 5 lesions were enhanced in a patchy or nodular manner; and 4 lesions exhibited irregular enhancement. All lesions exhibited clear boundaries with surrounding tissues but not smooth edges. Of the 105 cases, 84 lesions exhibited enhancement (Fig. 3), presenting as peripheral in early phase and center in late phase (Fig. 4).

As presented in Table IV, the mean ADC was $1.3 \pm 0.19 \times 10^{-3} \mathrm{~mm}^{2} / \mathrm{secforMDE}$ and was $1.03 \pm 0.32 \times 10^{-3} \mathrm{~mm}^{2} / \mathrm{sec}$ for breast cancer. There were significant differences in ADC between the two groups $(\mathrm{P}<0.001)$. 

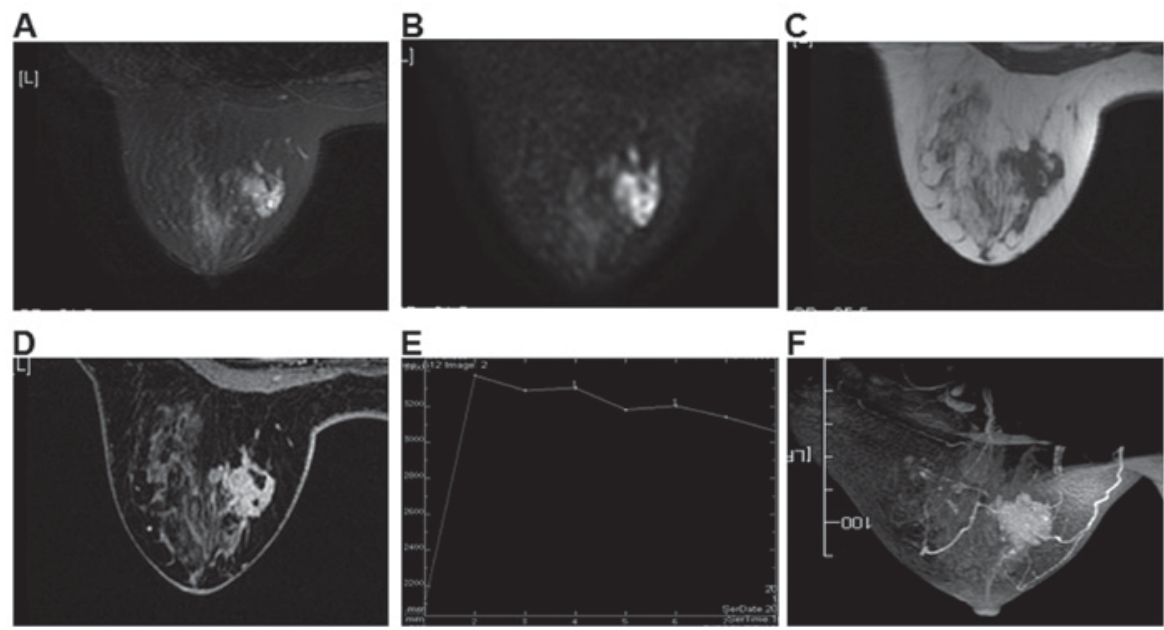

Figure 3. MRI features of breast cancer. (A) Heterogeneity of high signal in short tau inversion recovery sequence in left breast. (B) Diffusion weighted imaging sequence revealed heterogeneity of high signal. (C) T1 fast spin echo sequence revealed heterogeneity of low signal. (D) Dynamic contrast-enhanced MRI revealed that the lesion was unevenly enhanced with clear border in irregular form. (E) Time-signal intensity curve was type III. (F) MRI angiography identified coarse vessels around the lesion. MRI diagnosed breast cancer while pathology diagnosed invasive ductal carcinoma. MRI, magnetic resonance imaging.
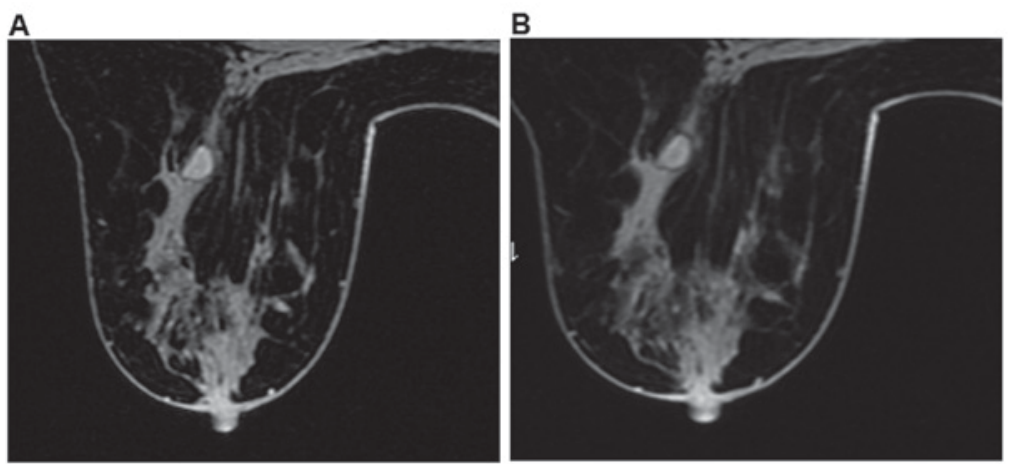

Figure 4. DCE-MRI features of breast cancer. (A) DCE-MRI revealed the rim of the lesion was enhanced circularly in the early phase and the center area was not enhanced. (B) The center was enhanced slowly in the delayed phase. DCE-MRI, dynamic contrast-enhanced magnetic resonance imaging.

For patients with MDE, the mean of EER, PER and $\mathrm{T}_{\text {peak }}$ was $0.67 \pm 0.16,2.59 \pm 0.46$ and $248 \pm 37$ sec, respectively; for patients with breast cancer, the mean was $1.03 \pm 0.40$, $1.08 \pm 0.40$ and $169 \pm 63 \mathrm{sec}$, respectively. There were significant differences in EER, PER and T peak between the two groups $(\mathrm{P}<0.001)$.

There were 28 lesions of type I, 5 of type II and 2 of type III in TIC for MDE. There were 3 lesions of type I, 47 of type II and 55 of type III for breast cancer. There were significant differences of TIC stages between the two groups $(\mathrm{P}<0.001)$.

Diagnostic efficiency of US, MRI and US with MRI. To determine the diagnostic values, diagnostic efficiency of US, MRI and US with MRI were compared. As presented in Table V, for the 35 patients with MDE, 30 were finally diagnosed with MDE, 3 were finally diagnosed with intraductal papilloma (IDP) and 2 were finally diagnosed with breast cancer by US (Figs. 5 and 6). For patients with breast cancer, 99/105 patients were finally diagnosed with breast cancer by US. In total, 1 patient with invasive ductal carcinoma (IDC) was misdiagnosed with intraductal papilloma (Fig. 7) by US; 2 patients with invasive ductal carcinoma were misdiagnosed as MDE; and
3 patients with intraductal carcinoma were misdiagnosed as inflammatory lesion, tumor-like hyperplasia and hyperplastic nodules, respectively.

Of the 35 patients with MDE, 32 were finally diagnosed as MDE, 1 was diagnosed as IDP and 2 were diagnosed as breast cancer by MRI. For breast cancer patients, 101/105 patients were finally diagnosed as breast cancer by MRI. In total, 1 IDC patient was misdiagnosed with intraductal papilloma; 2 patients with invasive ductal carcinoma were misdiagnosed with MDE; and 1 patient with intraductal carcinoma was misdiagnosed with tumor-like hyperplasia.

Of the 35 patients with MDE, 33 were finally diagnosed with MDE, 1 was diagnosed with IDP and 1 was diagnosed with breast cancer by US and MRI. For patients with clinically suspected breast cancer, 102/105 patients were finally diagnosed with breast cancer by US and MRI. A single patient with IDC was misdiagnosed with intraductal papilloma. A pair of patients with invasive ductal carcinoma were misdiagnosed with MDE and fibroadenoma.

There were no differences in diagnostic efficiency among US, MRI and US with MRI ( $\mathrm{P}=0.103, \mathrm{P}=0.263$ and $\mathrm{P}=0.403$, respectively). 
Table IV. Measurement index of MRI for MDE and breast cancer.

\begin{tabular}{lcccc}
\hline Variables & ADC & EER & PER & T peak \\
\hline MDE & $1.3 \pm 0.19$ & $0.67 \pm 0.16$ & $2.59 \pm 0.46$ & $248 \pm 37$ \\
Breast cancer & $1.03 \pm 0.32$ & $1.03 \pm 0.40$ & $1.08 \pm 0.40$ & $169 \pm 63 \mathrm{~s}$ \\
$\mathrm{t}$ & -4.313 & 5.259 & -18.77 & -7.080 \\
P-value & $<0.001$ & $<0.001$ & 0.004 & $<0.001$ \\
\hline
\end{tabular}

MRI, magnetic resonance imaging; ADC, apparent diffusion coefficient;EER, early-stage enhancement ratio; PER, peak-of-enhancement ratio; MDE, mammary duct ectasia; $\mathrm{T}$, time.

Table V. Diagnostic efficiency of US, MRI and US with MRI.

\begin{tabular}{|c|c|c|c|c|c|c|}
\hline \multirow[b]{2}{*}{ Variables } & \multicolumn{2}{|c|}{ US } & \multicolumn{2}{|c|}{ MRI } & \multicolumn{2}{|c|}{$\begin{array}{c}\text { US } \\
\text { with MRI }\end{array}$} \\
\hline & Yes & No & Yes & No & Yes & No \\
\hline MDE & 30 & 5 & 32 & 3 & 33 & 2 \\
\hline Breast cancer & 99 & 6 & 101 & 4 & 102 & 3 \\
\hline$\chi^{2}$ value & 2.664 & & 1.253 & & 0.622 & \\
\hline P-value & 0.103 & & 0.263 & & 0.403 & \\
\hline
\end{tabular}

US, ultrasound; MRI, magnetic resonance imaging; MDE, mammary duct ectasia; yes, imaging and pathology results were consistent with each other; no, imaging and pathology results were inconsistent with each other.

\section{Discussion}

In previous years, the incidence rate of breast cancer has been increasing and there is a lack of effective preventive measures (15). In addition, it is difficult to cure, despite its high incidence (1). It has been demonstrated that the imaging features of MDE may be similar to those of breast cancer (2). It is therefore important to distinguish MDE from breast cancer (16).

The mean age of patients with MDE was 33.58 \pm 8.81 years in the present study, consistent with another report (17). However, the mean age of patients with breast cancer was $44.97 \pm 11.04$ years, inconsistent with Jemal et al (18) who demonstrated that breast cancer frequently occurred in patients at the age of 50-69 years; however, Labib et al (19) demonstrated that the mean age was $46.3 \pm 12.4$ years, similar to the present study.

The present study revealed that the morphology features, including aspect ratio, border, shape and microcalcification were significantly increased in breast cancer compared with MDE. Features including unclear border, irregular, spicular sign and microcalcification, were unspecific for breast cancer, consistent with the report by Yabuuchi et al (20). The features of MDE were of regular shape and clear border, similar to other benign tumors.

CDFI grading may not be used to distinguish breast cancer and MDE. The RI and elastography score are useful in the differential diagnosis between breast cancer and MDE (9). It is reported that CDFI grading of II or III may be specific in diagnosing the malignancy of breast cancer (21-23). Stanzani et al (24) reported that the sensitivity and specificity were 72 and $67 \%$ in benign and malignant RI, respectively (threshold value, 0.75). Although the RI of benignancy and malignancy may overlap and the threshold value differs among studies, it was useful to distinguish the benign and malignant tumors $(24,25)$.

Gong et al (26) reported that UE may be as useful as traditional US, with sensitivity, specificity and accuracy of $92.65,73.39$ and $81.25 \%$, respectively. Combined with UE and US, the specificity and accuracy could increase to 95.97 and $93.23 \%$. Fischer et al (27) demonstrated that UE could be used to distinguish the breast cancer lesions with BI-RADS of 3-4, small diameter $(<2 \mathrm{~cm})$ and nontypical morphology. In the present study, it was revealed that UE was important to improve the specificity and accuracy of diagnosis, consistent with previous studies $(6,28)$. Cho et al (29) also supported that UE was important in the differential diagnosis of benign and malignant tumors.

Caivano et al (30) demonstrated that the ADC value of benign and malignant were $2.06 \pm 0.19 \times 10^{-3} \mathrm{~mm}^{2} / \mathrm{sec}$ and $1.03 \pm 0.07 \times 10^{-3} \mathrm{~mm}^{2} / \mathrm{sec}$, respectively. In the present study, the ADC was $1.3 \pm 0.19 \times 10^{-3} \mathrm{~mm}^{2} / \mathrm{sec}$ for MDE, decreased compared with that of the benign tumor and ADC was $1.03 \pm 0.32 \times 10^{-3} \mathrm{~mm}^{2} / \mathrm{sec}$ for breast cancer, within the range of that of the malignant tumors. Caivano et al (30) demonstrated that for benign tumors, the mean ADC value was $3.42 \pm 1.04 \times 10^{-3} \mathrm{~mm}^{2} / \mathrm{sec}$ for cysts, $1.57 \pm 0.78 \times 10^{-3} \mathrm{~mm}^{2} / \mathrm{sec}$ for fibroadenomas, $0.81 \pm 0.13 \times 10^{-3} \mathrm{~mm}^{2} / \mathrm{sec}$ for mammitis and $1.32 \pm 0.11 \times 10^{-3} \mathrm{~mm}^{2} / \mathrm{sec}$ fortumorwithout fluid, which wasconsistent with the present study (mean ADC, $1.3 \pm 0.19 \times 10^{-3} \mathrm{~mm}^{2} / \mathrm{sec}$ for MDE and 1.32 $\pm 0.11 \times 10^{-3} \mathrm{~mm}^{2} / \mathrm{sec}$ for tumor). Luo et al (31) demonstrated that the sensitivity and specificity of ADC were 74.1 and $70.3 \%$ respectively, with a threshold value of $1.2 \times 10^{-3} \mathrm{~mm}^{2} / \mathrm{sec}$. Yabuuchi et al (20) demonstrated that sensitivity, specificity, accuracy, positive and negative predictive value were $92,86,97,71$ and $91 \%$ respectively, with a threshold value of $1.1 \times 10^{-3} \mathrm{~mm}^{2} / \mathrm{sec}$, which were important indicators for diagnosis and differential diagnosis. In the present study there was a significant difference in ADC between MDE and breast cancer groups (threshold value, $1.2 \times 10^{-3} \mathrm{~mm}^{2} / \mathrm{sec}$ ), indicating the important function of ADC in the differential diagnosis.

A previous study demonstrated that the enhancement features of breast cancer were ring-like, with the rim of the lesion enhanced in the early phase, then moving to the center (32). The present study revealed that the enhancement in MDE (26/35) was ring-like or duct-like with thickening of the pipe wall, while the center was not enhanced in the delayed phase. Enhancement patterns were distinct between breast cancer and MDE; when the lesion was enhanced in a ring-like manner, the enhancement and timing of the lesion center should be observed.

Luo et al (31) demonstrated that the EER was increased and PER was decreased in malignant tumors compared with that in benign tumors. In the present study, EER was $1.03 \pm 0.40$ in the breast cancer group and $0.67 \pm 0.16$ in the MDE group, while PER was $1.08 \pm 0.40$ in the breast cancer group and $2.59 \pm 0.46$ in the MDE group, consistent with Luo's data (31). 
A

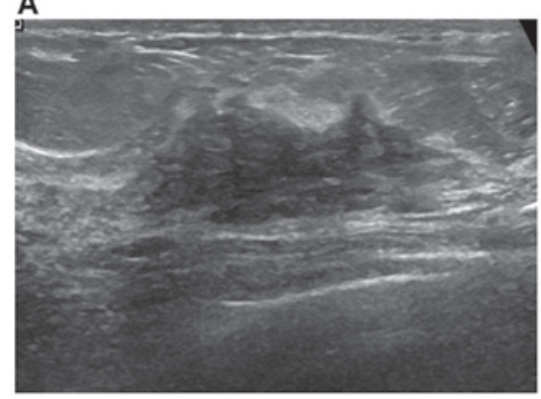

B

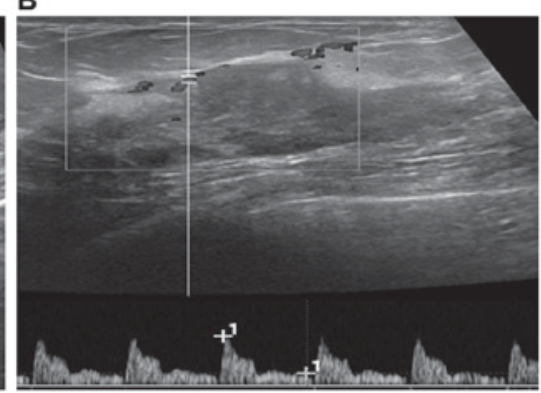

Figure 5. MDE misdiagnosed as breast cancer by US. (A) US revealed an even hypo echo mass under the right nipple with clear border in irregular form. The posterior margin was close to the pectoralis major but had not invaded. (B) Color Doppler flow imaging identified a weak blood signal around the mass. The resistance index was 0.74 . US diagnosed breast cancer, however pathology diagnosed MDE with acute and chronic inflammation. MDE, mammary duct ectasia; US, ultrasound.
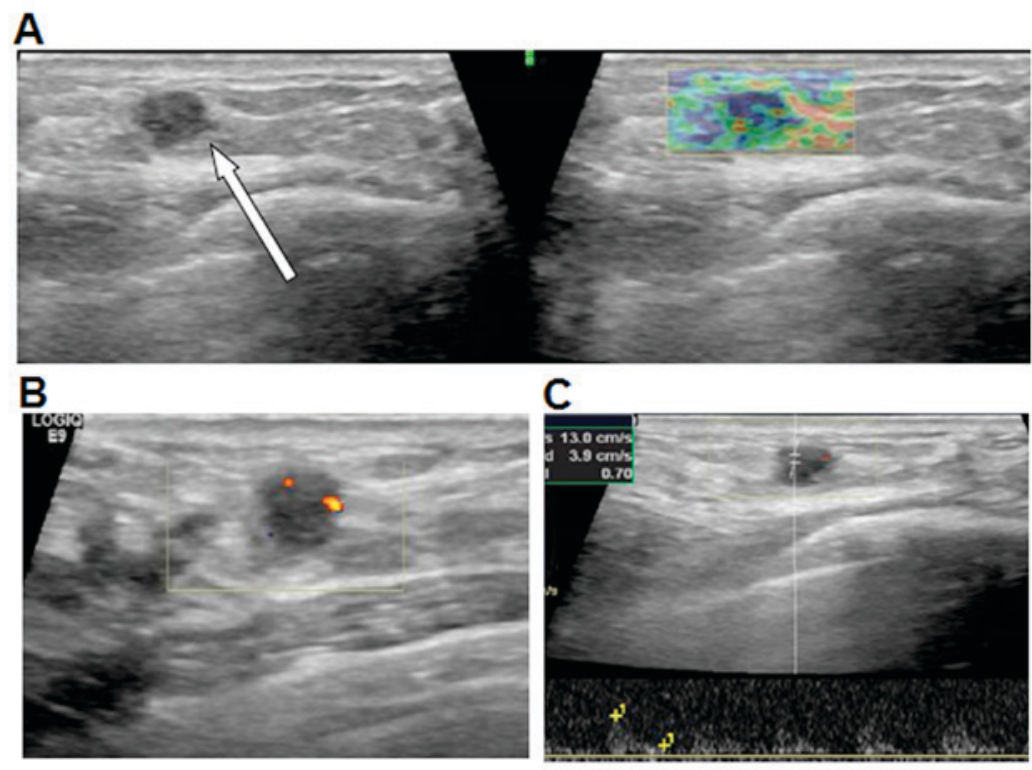

Figure 6. MDE misdiagnosed as breast cancer by US. (A) US identified a hypo echo nodule with unclear border and sharp corner in irregular form (arrow). US elastography scored 3 points. (B) Color Doppler flow imaging identified a dotted blood signal. (C) Resistance index was 0.7. US diagnosed breast cancer; pathology identified MDE. MDE, mammary duct ectasia; US, ultrasound.

Yuan et al (33) reported that the earlier the peak time, the greater the likelihood of malignancy. If peak time was $<180 \mathrm{sec}$, all the lesions may be malignant; if peak time was $180-270 \mathrm{sec}$, the malignancy rate was $85.7 \%$; if peak time was $>270 \mathrm{sec}$, all the lesions were benign. Sensitivity and specificity of peak time (cut-off value, $270 \mathrm{sec}$ ) were 95.83 and $92.30 \%$, respectively. The mean $\mathrm{T}_{\text {peak }}$ of breast cancer was $169 \pm 63 \mathrm{sec}$, consistent with the malignancy of $<180 \mathrm{sec}$. The mean $\mathrm{T}_{\text {peak }}$ of MDE was $248 \pm 37$ sec, ranging between 180-270 sec, exhibiting benign enhancement in the early phase. Fernández-Guinea et al (34) demonstrated that the nodules that were $<2 \mathrm{~cm}$ in diameter were enhanced homogeneously with obscure boundary, increased microvascular density and spicular sign compared with those that were smooth and clear. $\mathrm{T}_{\text {peak }}$ time $<2$ min indicated the association of more vessels compared with $\mathrm{T}_{\text {peak }}$ time $>2$ min. The lesions with PER $<150 \%$ were larger compared with those with PER $>150 \%$. PER in the present study was consistent with the results of Fernández-Guinea et al (34). Peak intensity was associated with the degree of vessel formation. In Fernández-Guinea et al (34), the indexes had an advantage in recognizing the malignant lesions, including unclear boundary or spicular, uneven or ring-like enhancement, type III TIC with diagnosis rates of 75, 81.2 and $57.4 \%$, respectively; in the present study, the rates were 92.4, 85.6 and $52.4 \%$, respectively.

It has been reported (31) that the majority of benign lesions belonged to type I TIC and malignant lesions presented type II and III, which was consistent with the results of the present study. In the MDE group, 28/35 (80\%) cases were type I and $7 / 35$ (20\%) cases were type II and III; in the breast cancer group, $3 / 105$ cases (2.9\%) were type I and 102/105 (97.1\%) were type II and III. Brookes et al (35) reported that intraductal papilloma was enhanced peripherally and then the center was enhanced. The majority of the intraductal papillomas were type II and III, similar to invasive ductal carcinoma (36), thus it was difficult to distinguish. Yuan et al (33) demonstrated that TIC pattern and $\mathrm{T}_{\text {peak }}$ were the most valuable factors for differential diagnosis.

Analyzing multiple parameters identified that US and MRI together may decrease the rate of misdiagnosis. There were 4 cases misdiagnosed as IDP, including 3 cases of MDE and 1 
A

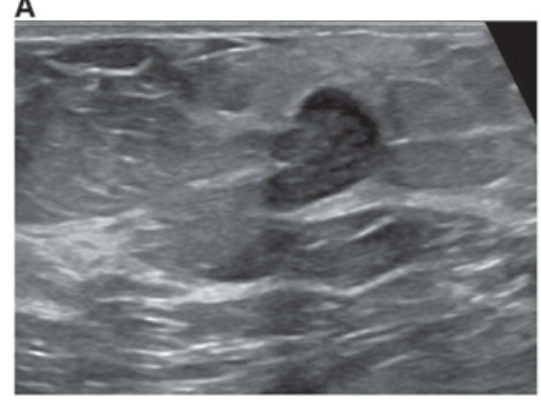

B

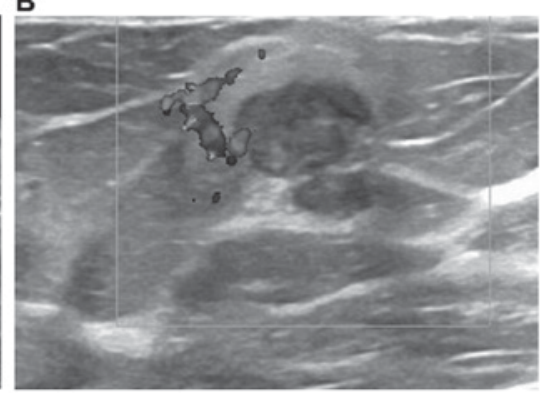

Figure 7. Invasive ductal carcinoma misdiagnosed as intraductal papilloma using US. (A) Solid nodules and dilated duct under the right nipple with smooth and clear pipe wall. (B) Color Doppler flow imaging identified no blood signal. US diagnosed intraductal papilloma. US, ultrasound.

A

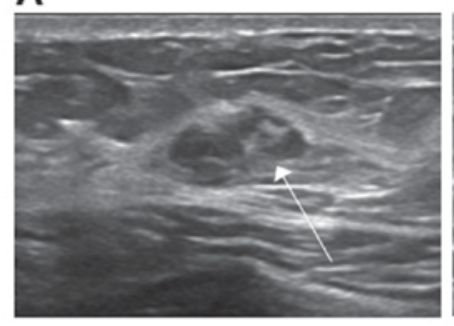

D

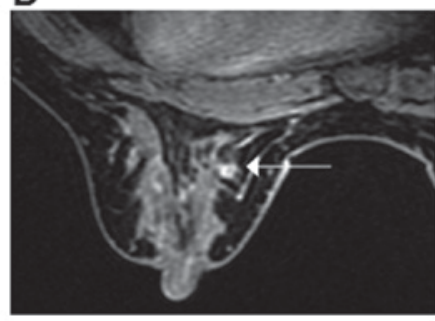

B

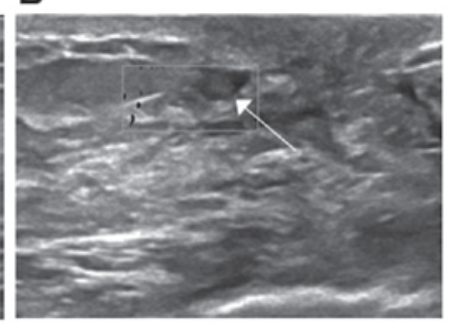

E

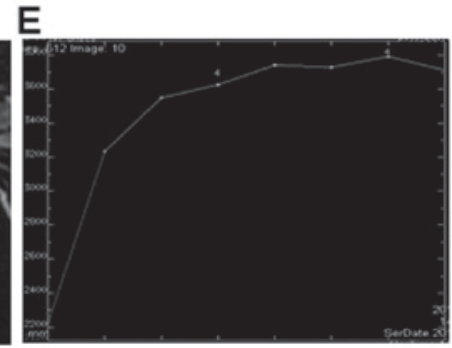

C
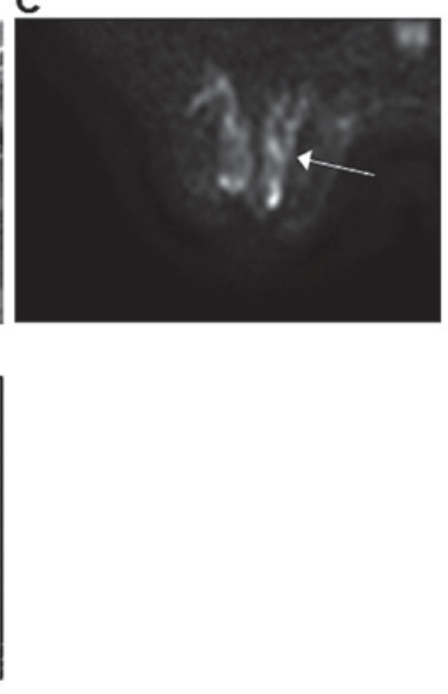

\begin{abstract}
Figure 8. Intraductal papilloma diagnosed using US with MRI. (A) US identified a hyper echo nodule with dilated duct above the nipple (arrow). (B) US identified a hyper echo nodule with dilated duct under the nipple (arrow) and clear border. Color Doppler flow imaging identified no blood signal. US diagnosed intraductal papilloma. (C) MRI diffusion weighted imaging sequencing revealed a high signal nodule with dilated duct (arrow). (D) Dynamic contrasted-enhanced MRI identified that the lesion was enhanced asymmetrically with clear border (arrow). (E) Time-signal intensity curve was type II. Pathology diagnosed intraductal papillary carcinoma. US, ultrasound; MRI, magnetic resonance imaging.
\end{abstract}

case of breast cancer. This was due to the IDP features being similar to those of MDE (type II), presenting with cyst-and-solid mass. US revealed a circle or oval solid echo with dotted or branched blood signals in dilated duct with a clear border and regular form (Fig. 8A and B). The majority of cases of MDE were located beneath the mammary areola, together with nipple retraction, sinus tract and fistula. The majority of IDP cases may attack the breast duct at level II or III. The predilection site may assist the diagnosis.

MRI identified the dotted, cord-like or small piece of low signal nodular (Fig. 8C and D) scattered following the duct and a spicule sign was seldom observed. The mean ADC value of IDP was between the value of normal breast and breast cancer. Woodhams et al (37) demonstrated that the mean ADC of IDP was $1.32 \pm 0.15 \times 10^{-3} \mathrm{~mm}^{2} / \mathrm{sec}$. In the MDE group, the ADC of MDE $\left(1.3 \pm 0.19 \times 10^{-3} \mathrm{~mm}^{2} / \mathrm{sec}\right)$ were similar. Brookes et al (35) revealed that IDP was benign with a rich blood supply that may be enhanced in the early phase with an enhancement rate decreased compared with breast cancer. The majority of TIC was type II and the minority were type III, similar to breast cancer (Fig. 8E). The underlying reasons may be as follows: i) IDP typically locates in the lactiferous sinus of the big latex duct near the nipple, which is supplied with rich blood; ii) blood is piled up in the latex duct; and iii) increased sensitivity and decreased specificity of MRI (37), consistent with the present study. IDP, MDE and breast cancer may all present as typeIIof TIC, therefore, TIC may not be used for differential diagnosis without other indicators.

The present study did not identify any significant differences in the diagnostic efficiency of MDE and breast cancer with US, MRI and US with MRI, which suggested that these three methods were equally efficient. Although multiple quantitative parameters and morphology alterations in US with MRI may still misdiagnose MDE and breast cancer, combining US and MRI may decrease the misdiagnosis rate. Therefore, further studies may use US and MRI in the diagnosis of MDE and breast cancer.

\section{Acknowledgements}

The present study was supported by The Shandong Provincial Science and Technology Development Program (grant 
no. 2014GSF118139) and the Shandong Major Development Program (grant no. 2016GSF201130).

\section{References}

1. Rahal RM, de Freitas-Júnior R, Carlos da Cunha L, Moreira MA, Rosa VD and Conde DM: Mammary duct ectasia: An overview. Breast J 17: 694-695, 2011

2. Kim BS, Lee JH, Kim WJ, Kim DC, Shin S, Kwon HJ, Park JS and Park YM: Periductal mastitis mimicking breast cancer in a male breast. Clin Imaging 37: 574-576, 2013.

3. Duchesne N, Skolnik S and Bilmer S: Ultrasound appearance of chronic mammary duct ectasia. Can Assoc Radiol J 56: 297-300, 2005.

4. Masciadri N and Ferranti C: Benign breast lesions: Ultrasound. J Ultrasound 14: 55-65, 2011.

5. Yamauchi $\mathrm{H}$, Woodward WA, Valero V, Alvarez $\mathrm{RH}$ Lucci A, Buchholz TA, Iwamoto T, Krishnamurthy S, Yang W, Reuben JM, et al: Inflammatory breast cancer: What we know and what we need to learn. Oncologist 17: 891-899, 2012.

6. Alhabshi SM, Rahmat K, Abdul Halim N, Aziz S, Radhika S, Gan GC, Vijayananthan A, Westerhout CJ, Mohd-Shah MN, Jaszle S, et al: Semi-quantitative and qualitative assessment of breast ultrasound elastography in differentiating between malignant and benign lesions. Ultrasound Med Biol 39: 568-578, 2013.

7. Suppiah S, Rahmat K, Rozalli FI and Azlan CA: Re: Improved diagnostic accuracy in differentiating malignant and benign lesions using single-voxel proton MRS of the breast at 3 T MRI. A reply. Clin Radiol 69: e110-e111, 2014.

8. Min Q, Shao K, Zhai L, Liu W, Zhu C, Yuan L and Yang J: Differential diagnosis of benign and malignant breast masses using diffusion-weighted magnetic resonance imaging. World J Surg Oncol 13: 32, 2015.

9. Zhang F, Yu D, Guo M, Wang Q, Yu Z, Zhou F, Zhao M, Xue F and Shao G: Ultrasound elastography and magnetic resonance examinations are effective for the accurate diagnosis of mammary duct ectasia. Int J Clin Exp Med 8: 8506-8515, 2015.

10. Hsu HH, Yu JC, Hsu GC, Chang WC, Yu CP, Tung HJ, Tzao C and Huang GS: Ultrasonographic alterations associated with the dilatation of mammary ducts: Feature analysis and BI-RADS assessment. Eur Radiol 20: 293-302, 2010.

11. Itoh A, Ueno E, Tohno E, Kamma H, Takahashi H, Shiina T, Yamakawa $\mathrm{M}$ and Matsumura T: Breast disease: Clinical application of US elastography for diagnosis. Radiology 239: 341-350 2006.

12. Goddi A, Bonardi M and Alessi S: Breast elastography: A literature review. J Ultrasound 15: 192-198, 2012.

13. Radiology ACo: Breast imaging reporting and data system (BI-RADS), 4th edition. Reston, VA: American College of Radiology 2003.

14. El Khouli RH, Macura KJ, Jacobs MA, Khalil TH, Kamel IR, Dwyer A and Bluemke DA: Dynamic contrast-enhanced MRI of the breast: Quantitative method for kinetic curve type assessment. AJR Am J Roentgenol 193: W295-W300, 2009.

15. Howell A, Anderson AS, Clarke RB, Duffy SW, Evans DG, Garcia-Closas M, Gescher AJ, Key TJ, Saxton JM and Harvie MN: Risk determination and prevention of breast cancer. Breast Cancer Res 16: 446, 2014.

16. Holley SO, Appleton CM, Farria DM, Reichert VC, Warrick J, Allred DC and Monsees BS: Pathologic outcomes of nonmalignant papillary breast lesions diagnosed at imaging-guided core needle biopsy. Radiology 265: 379-384, 2012

17. Cheng J, Ding HY and DU YT: Granulomatous lobular mastitis associated with mammary duct ectasia: A clinicopathologic study of 32 cases with review of literature. Zhonghua Bing Li Xue Za Zhi 42: 665-668, 2013 (In Chinese).

18. Jemal A, Ward E and Thun MJ: Recent trends in breast cancer incidence rates by age and tumor characteristics among U.S women. Breast Cancer Res 9: R28, 2007.

19. Labib NA, Ghobashi MM, Moneer MM, Helal MH and Abdalgaleel SA: Evaluation of BreastLight as a tool for early detection of breast lesions among females attending national cancer institute, Cairo University. Asian Pac J Cancer Prev 14: 4647-4650, 2013.
20. Yabuuchi H, Matsuo Y, Okafuji T, Kamitani T, Soeda H, Setoguchi T, Sakai S, Hatakenaka M, Kubo M, Sadanaga N, et al: Enhanced mass on contrast-enhanced breast MR imaging: Lesion characterization using combination of dynamic contrast-enhanced and diffusion-weighted MR images. J Magn Reson Imaging 28: 1157-1165, 2008.

21. Li B, Zhao X, Dai SC and Cheng W: Associations between mammography and ultrasound imaging features and molecular characteristics of triple-negative breast cancer. Asian Pac J Cancer Prev 15: 3555-3559, 2014.

22. Zeng H, Zhao YL, Huang Y, Lin X, Chen XY and Li AH: Values of color doppler flow imaging and imaging changes of breast fascia and ligament in differential diagnosis of small breast neoplasms. Ai Zheng 25: 339-342, 2006 (In Chinese).

23. Yang M, Liu F, Gu XN, Cai YL, Wang YY and Zhou WJ: The application value of BI-RADS lexicon and high-frequency CDFI scoring in differentiation of benign from malignant lesions of the breast. Zhonghua Yi Xue Za Zhi 93: 1833-1835, 2013 (In Chinese)

24. Stanzani D, Chala LF, Barros Nd, Cerri GG and Chammas MC Can Doppler or contrast-enhanced ultrasound analysis add diagnostically important information about the nature of breast lesions? Clinics (Sao Paulo) 69: 87-92, 2014.

25. del Cura JL, Elizagaray E, Zabala R, Legórburu A and Grande D: The use of unenhanced Doppler sonography in the evaluation of solid breast lesions. AJR Am J Roentgenol 184: 1788-1794, 2005.

26. Gong X, Wang Y and Xu P: Application of real-time ultrasound elastography for differential diagnosis of breast tumors. J Ultrasound Med 32: 2171-2176, 2013.

27. Fischer T, Sack I and Thomas A: Characterization of focal breast lesions by means of elastography. Rofo 185: 816-823, 2013.

28. Hassan HHM, Zahran MHM, Hassan HEP, Abdel-Hamid AEM and Fadaly GAS: Diffusion magnetic resonance imaging of breast lesions: Initial experience at Alexandria University. Alex J Med 49: 265-272, 2013

29. Cho N, Jang M, Lyou CY, Park JS, Choi HY and Moon WK: Distinguishing benign from malignant masses at breast US: Combined US elastography and color doppler US-influence on radiologist accuracy. Radiology 262: 80-90, 2012.

30. Caivano R, Villonio A, D' Antuono F, Gioioso M, Rabasco P, Iannelli G, Zandolino A, Lotumolo A, Dinardo G, Macarini L, et al: Diffusion weighted imaging and apparent diffusion coefficient in 3 tesla magnetic resonance imaging of breast lesions. Cancer Invest 33: 159-164, 2015.

31. Luo Y, Yu J, Chen D, Xu Z and Zeng H: The actions of diffusion weighted imaging (DWI) and dynamic contrast enhanced MRI in differentiating breast tumors. Sheng Wu Yi Xue Gong Cheng Xue Za Zhi 30: 1219-1223, 2013 (In Chinese)

32. Yeh ED, Slanetz PJ, Edmister WB, Talele A, Monticciolo D and Kopans DB: Invasive lobular carcinoma: Spectrum of enhancement and morphology on magnetic resonance imaging. Breast J 9: 13-18, 2003.

33. Yuan HM, Yu JQ, Chu ZG and Peng LQ: Distinguishing benign and malignant lesions with time-signal intensity curve of dynamic contrast-enhanced breast MRI scanning. Sichuan Da Xue Xue Bao Yi Xue Ban 42: 556-559, 2011 (In Chinese).

34. Fernández-Guinea O, Andicoechea A, González LO, González-Reyes S, Merino AM, Hernández LC, López-Muñiz A, García-Pravia P and Vizoso FJ: Relationship between morphological features and kinetic patterns of enhancement of the dynamic breast magnetic resonance imaging and clinico-pathological and biological factors in invasive breast cancer. BMC Cancer 10: 8, 2010.

35. Brookes MJ and Bourke AG: Radiological appearances of papillary breast lesions. Clin Radiol 63: 1265-1273, 2008

36. Zhu Y, Zhang S, Liu P, Lu H, Xu Y and Yang WT: Solitary intraductal papillomas of the breast: MRI features and differentiation from small invasive ductal carcinomas. AJR Am J Roentgenol 199: 936-942, 2012.

37. Woodhams R, Matsunaga K, Kan S, Hata H, Ozaki M, Iwabuchi K, Kuranami M, Watanabe M and Hayakawa K: ADC mapping of benign and malignant breast tumors. Magn Reson Med Sci 4: 35-42, 2005

This work is licensed under a Creative Commons Attribution-NonCommercial-NoDerivatives 4.0 International (CC BY-NC-ND 4.0) License. 